ALEA, Lat. Am. J. Probab. Math. Stat. 18, 945-962 (2021)

DOI: 10.30757/ALEA.v18-34

\title{
Ballistic random walks in random environment as rough paths: convergence and area anomaly
}

\section{Olga Lopusanschi and Tal Orenshtein}

TU Berlin and WIAS Berlin

Mohrenstraße 3910117 Berlin, Germany.

E-mail address: orenshtein@wias-berlin.de

\begin{abstract}
Annealed functional CLT in the rough path topology is proved for the standard class of ballistic random walks in random environment. Moreover, the 'area anomaly', i.e. a deterministic linear correction for the second level iterated integral of the rescaled path, is identified in terms of a stochastic area on a regeneration interval. The main theorem is formulated in more general settings, namely for any discrete process with uniformly bounded increments which admits a regeneration structure where the regeneration times have finite moments. Here the largest finite moment translates into the degree of regularity of the rough path topology. In particular, the convergence holds in the $\alpha$-Hölder rough path topology for all $\alpha<1 / 2$ whenever all moments are finite, which is the case for the class of ballistic random walks in random environment. The latter may be compared to a special class of random walks in Dirichlet environments for which the regularity $\alpha<1 / 2$ is bounded away from $1 / 2$, explicitly in terms of the corresponding trap parameter.
\end{abstract}

\section{Introduction}

Rough path theory has been extensively developing since it was introduced by Lyons (1998). The theory provides a framework to solutions to SDEs driven by non-regular signals such as Brownian motions, while keeping the solution map continuous with respect to the signal. The Itô theory of stochastic integration, being an $L^{2}$ theory in essence, does not allow integration path-by-path, and hence does not give rise to solutions with such continuity property.

As it was observed by Lyons, the difficulty is not only a technical issue; in any separable Banach space $\mathcal{B} \subset \mathcal{C}[0,1]$ containing the sample paths of Brownian

Received by the editors August 6th, 2020; accepted February 10th, 2021.

2010 Mathematics Subject Classification. 60K05, 60K37, 60K40,60L20, 82B41.

Key words and phrases. Levy area, rough paths, annealed invariance principles, area anomaly, random walks in random environment, ballisticity conditions, regeneration structure.

The research of T.O. was supported by the German Research Foundation through the research unit FOR 2402 - Rough paths, stochastic partial differential equations and related topics. 
motions a.s. the map $(f, g) \rightarrow \int_{0}^{\cdot} f(t) \dot{g}(t) \mathrm{d} t$ defined on smooth maps cannot be extended to a continuous map on $\mathcal{B} \times \mathcal{B}$ (see Friz and Hairer, 2014, Proposition 1.1 and the references therein). Some additional information on the path is needed to achieve continuity, namely the so called "iterated integrals", where the number of iterations needed is determined by the regularity of the signal.

Fix $T>0$ and $X:[0, T] \rightarrow \mathbb{R}^{d}$. The $M$-th level iterated integral of $X$ is

$$
S_{s, t}^{M}(X)=\int_{s<u_{1}<\ldots<u_{M}<t} \mathrm{~d} X_{u_{1}} \cdots \mathrm{d} X_{u_{M}}, s<t, s, t \in[0, T] .
$$

Note that the definition of iterated integrals assumes a notion of integration with respect to $X$.

Lyons' theory uses the information coming from the iterated integral as a postulated high level information and constructs a space (called the rough path space) in which solutions to SDEs driven by Brownian motion are continuous with respect to the latter. In this case two levels of iteration are enough since the Brownian motion is $\alpha$-Hölder for some $\alpha>\frac{1}{3}$ (and actually for all $\alpha<\frac{1}{2}$ ). More generally, roughly speaking, in case the signal is $\alpha$-Hölder continuous for some $\alpha \in(0,1]$, then $M=\lfloor 1 / \alpha\rfloor$ levels of iteration are sufficient (and necessary).

For discrete processes with regeneration structure such as ballistic random walks in random environment (RWRE), invariance principles are well known. The main result of this paper, Theorem 3.3, shows that after lifting the path we have as well a scaling limit in the rough path topology where the regularity is determined by the moments of the regenerations.

The application to the so-called ballistic RWREs, formulated in Theorem 5.3, is then immediate, and since regeneration times have all moments Sznitman (2000) the convergence in spaces of regularity $\alpha$ is taken all the way to $\alpha<\frac{1}{2}$. The theorem is also applied to random walks in Dirichlet environments with large enough trap parameter, where in this case the convergence is on a limited regularity space, see Theorem 5.5 for the precise statement.

When a scaling limit is known for some process in the uniform topology, one might be interested to get a richer information about the limit. For inhomogeneous random walks with regeneration structure, an interesting phenomenon yields. As it turns out, unlike the "classical" invariance principles, when considering the second level iterated integral, which is related to the running signed area of the process as we show, the local fluctuations do not disappear in the limit, and a correction has to be considered. Moreover, thanks to the i.i.d structure of the walk on regeneration intervals, the law of large numbers allows us to write the correction as a linear function in time $(t \Gamma)_{0 \leq t \leq T}$, called the area anomaly. In particular, $\Gamma$ is a deterministic matrix which is the expected signed area accumulated in a regeneration interval, divided by its expected length, see the main result, Theorem 3.3.

Another application is related to the Wong-Zakai type approximations of solutions to SDEs. Let $\left(B^{N}\right)_{N}$ be a sequence of semimartingales converging weakly in the uniform topology to a Brownian motion $B$. An interesting question is to understand the approximating differential equations, where the noise is replaces by $B^{N}$. Let $X$ be a solution to a SDE with nice (in an appropriate sense) drift and diffusion coefficients and let $X^{N}$ be a solution to corresponding difference equation driven by $B^{N}$. The Wong-Zakai Theorem implies that it is not true in general that $X^{N}$ converges to $X$ whenever the convergence of the noise holds in the uniform topology Wong and Zakai (1965). However, if the weak convergence of $B^{N}$ to $B$ 
holds in the rough path space of regularity $\alpha$ with a linear area correction $t \Gamma$, for some $\alpha \in\left(\frac{1}{3}, \frac{1}{2}\right)$, then the answer is affirmative, where the SDE under consideration has to be modified by adding a drift term which is explicit in terms of $\Gamma$ Kelly (2016).

Other aspect of noise approximations effects is related to SPDEs. The theory of rough path was strengthened with Gubinelli's notion of controlled rough path (Gubinelli, 2004) and branched rough path (Gubinelli, 2010) which extend the notion of integration and of solutions to differential equation with respect to an abstract data coming from the noise. This then inspired Martin Hairer to develop the farreaching theory of regularity structures (Hairer, 2014), which is now extensively studied. A similar question is fundamental to SPDEs: what can be learned on the solutions if rather than mollifying the noise by a smooth function one takes more complicated approximations? For a recent progress in this direction, see Bruned et al. (2019).

Going back to the Brownian case, the fundamental result related to our work is the Donsker's invariance principle in the rough path topology (Breuillard et al., 2009). An extension to random walks with general covariances was proved in Kelly (2016).

In Lopusanschi and Simon $(2017,2018)$ the authors studied some discrete processes converging to Brownian motion in $\mathbb{R}^{d}$ in the rough path topology with area anomaly which was constructed explicitly. Our main idea of our proof is inspired by theirs, with two main differences. First, we do not use the strong Markov property for the excursions, which, for a finitely supported jump distribution implies that the excursions have exponential tail. Instead, we only assume i.i.d. regeneration structure and moments of the regeneration times. Second, the discrete processes in these papers are homogeneous in space (a simple random walk on periodic graphs, see Lopusanschi and Simon, 2017, or hidden Markov walk where the jumps are independent of the current position, see Lopusanschi and Simon, 2018). In our case we allow the process to have jump distributions that are inhomogeneous in space.

Another interesting example is a Brownian motion in magnetic field Friz et al. (2015). Here the discretization converges to an enhanced Brownian motion with an explicit area anomaly.

The problem of discrete processes seen as rough paths is dealt with in other context as well. Kelly (2016) and Kelly and Melbourne (2016), and the more recent Friz and Zhang (2018) used the rough path framework to deal with discrete approximations of SDEs. The case of random walks on nilpotent covering graphs was considered in Ishiwata et al. (2020a,b); Namba (2018) where the corresponding area anomaly is identified in terms of harmonic embeddings (cf. Ishiwata et al., 2020a, equation (2.6)). Anther paper concerning discrete processes which is of relevance here is Chevyrev and Friz (2019). In that paper the authors showed a general construction of rough SDEs allowing rough paths with jumps beyond linear interpolations.

1.1. Structure of the paper. In order to keep the paper as self-contained as possible in Chapter 2 we discuss basic notions in rough path theory and set up the framework to be used in the rest of the paper. In chapter 3 we formulate our main result, Theorem 3.3. In chapter 4 we give some simple examples of processes converging in the rough path topology and lacking or having non-zero area anomaly. In Chapter 5 
we present other special cases of our main result. Particular cases are ballistic random walks in random environment, for which we also present an open problem, and random walks in Dirichlet environments. Finally, in Chapter 6 we give the proof of Theorem 3.3.

\section{Basic notions in rough path theory}

The aim of this section is to introduce briefly the basic objects in our framework. These are adapted from Chapters 2 and 3 of Friz and Hairer (2014). The experienced reader can safely jump to Remark 2.4. Since we assume here no familiarity of the reader with rough path theory we added a short discussion after Proposition 2.3 which is somewhat loosely formulated and should be treated accordingly. For an extensive account of the theory the reader is suggested to consult Friz and Victoir (2010) and Friz and Hairer (2014).

Initially developed for solving differential equations, rough path theory is also useful in the discrete setting, and in particular for studying the convergence of discrete processes. For example, in the uniform topology a simple random walk (SRW) on $\mathbb{Z}^{2}$ to which we add deterministic four steps clockwise loops every two steps (see Figure 2.1 below) converges to the same Brownian motion as a SRW which stays still for four steps every two steps. Thus in the uniform topology the loops simply disappear at the limit.

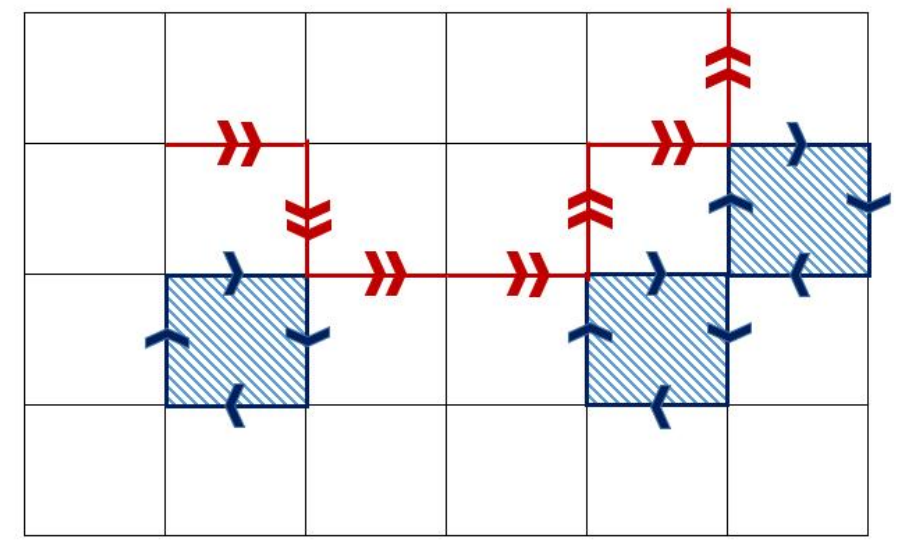

Figure 2.1. A simple random walk with a deterministic loop every two steps. The double arrows (in red) are the random walk's steps while the added loops are presented by the single arrows (in blue).

The loops certainly do not play a role if one is interested in the limit trajectory only. However, if one wishes to study more aspects of the limit, the accumulated area created by the loops could be also taken into consideration. A basic example for accumulated area in the continuous setting is provided by the "bubble areas" of Lejay (2003). This weakness of the uniform topology is precisely one of the problems that rough path theory palliates.

Following Friz and Victoir (2010) we denote by $\otimes$ two different actions: 
- for two elements of vector spaces, it is the usual tensor product: if $V$ and $W$ are $d$-dimensional, respectively $d^{\prime}$-dimensional vector spaces, for $v \in V$ and $w \in W, v \otimes w$ is the matrix $\left(v_{i} w_{j}\right)_{1 \leq i \leq d, 1 \leq j \leq d^{\prime}}$;

- for two elements of a group (in our case, $G^{2}\left(\mathbb{R}^{d}\right)$, defined below), it denotes the corresponding group operation.

The continuous process obtained by linear interpolation (or any other piecewise $C^{1}$ interpolation) of a discrete process, as well as its corresponding iterated integrals can be encoded in terms of elements of a particular nilpotent Lie group (see Friz and Hairer, 2014, Section 2.3 for more details). For simplicity and since our motivation in this paper is to prove convergence to Brownian motion, which is $\alpha$-Hölder for all $\alpha<1 / 2$, we adapt the general point of view taken in the book Friz and Hairer (2014) and consider (1.1) in the case $M \leq 2$, i.e. with only two levels of iteration. Therefore in the rest of the paper, we write $S_{s, t}(X)$ for $S_{s, t}^{2}(X)$. The pairs $\left(X_{s, t}, S_{s, t}(X)\right)$, $s<t$, with $X_{s, t}=X_{t}-X_{s}$, for a smooth path $X$, have a natural group structure with respect to increment concatenation. Here is the formal definition (see also the algebraic conditions in Proposition 2.4 for the corresponding formulation in terms of paths).

Definition 2.1 (The group $G^{2}\left(\mathbb{R}^{d}\right)$ ). The step-2 nilpotent Lie group $G^{2}\left(\mathbb{R}^{d}\right) \subset$ $\mathbb{R}^{d} \oplus\left(\mathbb{R}^{d}\right)^{\otimes 2}$ is defined as follows. An element can be presented by a pair $(a, b) \in$ $\mathbb{R}^{d} \times \mathbb{R}^{d \times d}$ (that is $a$ is a vector and $b$ is a matrix), the group operation $\otimes$ is defined by

$$
(a, b) \otimes\left(a^{\prime}, b^{\prime}\right)=\left(a+a^{\prime}, b+b^{\prime}+a \otimes a^{\prime}\right),
$$

and the following condition holds

$$
\forall(a, b) \in G^{2}\left(\mathbb{R}^{d}\right), \operatorname{Sym}(b)=\frac{1}{2} a \otimes a,
$$

where $\operatorname{Sym}(\cdot)$ is the symmetric part of an element, that is $\operatorname{Sym}(b)_{i, j}=\frac{1}{2}\left(b_{i, j}+b_{j, i}\right)$. (For clarity, we emphasize that above we used $a \otimes a^{\prime}=\left(a_{i} a_{j}^{\prime}\right)_{i, j}$ for the tensor product).

For an element $(a, b), a$ and $b$ are called the first and the second level, respectively.

The topology of $G^{2}\left(\mathbb{R}^{d}\right)$ is induced by the Carnot-Caratheodory norm $\|\cdot\|_{G^{2}\left(\mathbb{R}^{d}\right)}$, which gives for an element $(a, b) \in G^{2}\left(\mathbb{R}^{d}\right)$ the length of the shortest path with bounded variation that can be "encoded" as $(a, b)$, i.e. whose increment is $a$ and whose iterated integral is $b$. In other words

$$
\|(a, b)\|_{G^{2}\left(\mathbb{R}^{d}\right)}:=\inf \left\{\int_{0}^{1}|\dot{\gamma}(t)| \mathrm{d} t\right\},
$$

where the infimum is over all $\gamma:[0,1] \rightarrow \mathbb{R}^{d}$ of bounded variation so that $\left(\gamma_{0,1}, S_{0,1}(\gamma)\right)=(a, b)$. Showing that the set on which the infimum is taken is non-empty is a non-trivial statement and is the content of Chow's Theorem, see Friz and Victoir (2010, Theorem 7.28). The norm defined in this fashion induces a continuous metric $\mathbf{d}$ on $G^{2}\left(\mathbb{R}^{d}\right)$ through the application

$$
\begin{array}{ccc}
\mathbf{d}: \quad G^{2}\left(\mathbb{R}^{d}\right) \times G^{2}\left(\mathbb{R}^{d}\right) & \rightarrow & \mathbb{R}_{+} \\
(g, h) & \mapsto & \left\|g^{-1} \otimes h\right\|_{G^{2}\left(\mathbb{R}^{d}\right)} .
\end{array}
$$

$\left(G^{2}\left(\mathbb{R}^{d}\right), \mathbf{d}\right)$ is then a geodesic space, i.e. any two points can be connected by a geodesic. 
Definition 2.2 (Rough paths on $G^{2}\left(\mathbb{R}^{d}\right)$ ). Let $1 / 3<\alpha<1 / 2$. An $\alpha$-Hölder geometric rough path on $G^{2}\left(\mathbb{R}^{d}\right)$ is an element $\mathbf{X}=(X, \mathbb{X}) \in \mathcal{C}^{\alpha}\left([0, T], G^{2}\left(\mathbb{R}^{d}\right)\right)$. More preciesly, $\left(X_{s, t}, \mathbb{X}_{s, t}\right), s, t \in[0, T], s<t$, are in $G^{2}\left(\mathbb{R}^{d}\right)$ and the path is an $\alpha$-Hölder continuous function with respect to the distance $\mathbf{d}$.

Without going into details we remark that rough path theory also deals with rough paths which are not geometric, i.e., those for which (2.2) does not hold.

An example in the probabilistic setting of an $\alpha$-Hölder geometric rough path, for any $\alpha \in\left(\frac{1}{3}, \frac{1}{2}\right)$ is the Brownian motion rough path, which is also known as the enhanced Brownian motion. It is constructed using Stratonovich integration as follows:

$$
\left(B_{s, t}, \mathcal{S}_{s, t}\right)=\left(B_{t}-B_{s}, \int_{s \leq u<v \leq t} \circ d B_{u} \otimes \circ d B_{v}\right), 0 \leq s<t .
$$

The group structure on $G^{2}\left(\mathbb{R}^{d}\right)$ and the Carnot-Caratheodory norm and distance are particularly tamed for treating path concatenations. For example the norm is sub-additive. In particular, for a path $\mathbf{X}=(X, \mathbb{X})$ which takes value in $G^{2}\left(\mathbb{R}^{d}\right)$ let $\mathbf{X}_{s, t}:=\mathbf{X}_{s}^{-1} \otimes \mathbf{X}_{t}$. Then for every $s<u<t$

$$
\left\|\mathbf{X}_{s, t}\right\|_{G^{2}\left(\mathbb{R}^{d}\right)}=\left\|\mathbf{X}_{s, u} \otimes \mathbf{X}_{u, t}\right\|_{G^{2}\left(\mathbb{R}^{d}\right)} \leq\left\|\mathbf{X}_{s, u}\right\|_{G^{2}\left(\mathbb{R}^{d}\right)}+\left\|\mathbf{X}_{u, t}\right\|_{G^{2}\left(\mathbb{R}^{d}\right)} .
$$

The next proposition can found be useful for actual estimations. It leans on the equivalence

$$
C^{-1} \leq \frac{\|(a, b)\|_{G^{2}\left(\mathbb{R}^{d}\right)}}{|a|_{\mathbb{R}^{d}}+|b|_{\mathbb{R}^{d} \otimes \mathbb{R}^{d}}^{1 / 2}} \leq C,
$$

where $C \geq 1$ is a constant, $|\cdot|_{\mathbb{R}^{d}}$ is the Euclidean norm on $\mathbb{R}^{d}$ and $|\cdot|_{\mathbb{R}^{d} \otimes \mathbb{R}^{d}}$ is the induced matrix norm.

Assume that $\left(X_{s, t}, \mathbb{X}_{s, t}\right), 0 \leq s<t \leq T$, take value in $\mathbb{R}^{d} \times \mathbb{R}^{d \times d}$. Define

$$
\||(X, \mathbb{X})|\|_{\alpha}:=\|X\|_{\alpha}+\|\mathbb{X}\|_{2 \alpha}
$$

where

$$
\|X\|_{\alpha}=\sup _{s<t, s, t \in[0, T]} \frac{\left|X_{s, t}\right|_{\mathbb{R}^{d}}}{|t-s|^{\alpha}} \text { and }\|\mathbb{X}\|_{2 \alpha}=\sup _{s<t, s, t \in[0, T]} \frac{\left|\mathbb{X}_{s, t}\right|_{\mathbb{R}^{d} \otimes \mathbb{R}^{d}}}{|t-s|^{2 \alpha}} .
$$

Proposition 2.3. (Friz and Hairer, 2014, Proposition 2.4) Let $\alpha \in\left(\frac{1}{3}, \frac{1}{2}\right]$. $\mathbf{X}=(X, \mathbb{X}):\{0 \leq s<t \leq T\} \rightarrow \mathbb{R}^{d} \times \mathbb{R}^{d \times d}$ is a geometric rough path as in Definition 2.2 if and only if $X_{s, t}=X_{t}-X_{s}$ and the following assumptions hold:

- $\||(X, \mathbb{X})|\|_{\alpha}<\infty$.

- $\mathbb{X}_{s, t}=\mathbb{X}_{s, u}+\mathbb{X}_{u, t}+X_{s, u} \otimes X_{u, t}$ for every $s<u<t$ (Chen's relation).

- $\operatorname{Sym}\left(\mathbb{X}_{s, t}\right)=\frac{1}{2} X_{s, t} \otimes X_{s, t}$ for every $s<t$ (integration by parts property).

To end this brief review we mention an alternative definition of the group which has some nice interpretation in terms of signed area. A path $\mathbf{X}$ considered in Definition 2.2 has increments in $G^{2}\left(\mathbb{R}^{d}\right)$. This is relevant for the notion of integration, which is, roughly speaking, defined based on "sewing" according to the increments. However, since the symmetric part of the second level depends entirely on the first level by definition, to handle path increments the following alternative definition for the group on which the rough paths are considered is sometimes more useful. The corresponding antisymmetric group operation $\wedge$ is defined by

$$
(a, b) \wedge\left(a^{\prime}, b^{\prime}\right)=\left(a+a^{\prime}, b+b^{\prime}+\frac{1}{2}\left(a \otimes a^{\prime}-a^{\prime} \otimes a\right)\right) .
$$


In particular, unlike the case of the law $\otimes$ where an element $(a, b)$ represents a path with an increment $a$ and an iterated integral $b$, in the case of the antisymmetric product an element $(a, b)$ represents a path where $a$ is still an increment but $b$ is now the corresponding area. In other words, for $(X, \mathbb{X}) \in G^{2}\left(\mathbb{R}^{d}\right)$ we consider $(X, A)$ instead, where $A_{s, t}^{i, j}=\frac{1}{2}\left(\mathbb{X}_{s, t}^{i, j}-\mathbb{X}_{s, t}^{j, i}\right)$.

For example, the Brownian motion considered as a rough path in the case of the antisymmetric product $\wedge$ has the form

$$
\mathbb{B}_{s, t}^{\wedge}=\left(B_{t}-B_{s}, \mathcal{A}_{s, t}\right), 0 \leq s \leq t,
$$

where $\mathcal{A}$ is the stochastic signed area of $B$, called the Lévy area. One also remark that the Lévy area is invariant under performing the integration in either the Stratonovich or the Itô sense.

The operation defined in (2.8) has a geometric interpretation which shows why the group is suitable for concatenating paths. The first level translates into path concatenation, whereas the second one gives the law of the "area concatenation" (signed area of concatenated paths). Figure 2.2 demonstrates how to calculate the signed area of two concatenated curves. The areas of $\gamma_{1}, \gamma_{2}$ and that of the triangle (formed by the increments of $\gamma_{1}$ and $\gamma_{2}$ ) in the figure correspond respectively to $b$, $b^{\prime}$ and $\frac{1}{2}\left(a \otimes a^{\prime}-a^{\prime} \otimes a\right)$ in formula (2.8). This rule for the area of concatenated paths is also commonly referred as the Chen's rule. It plays a fundamental role in the theory of rough paths.

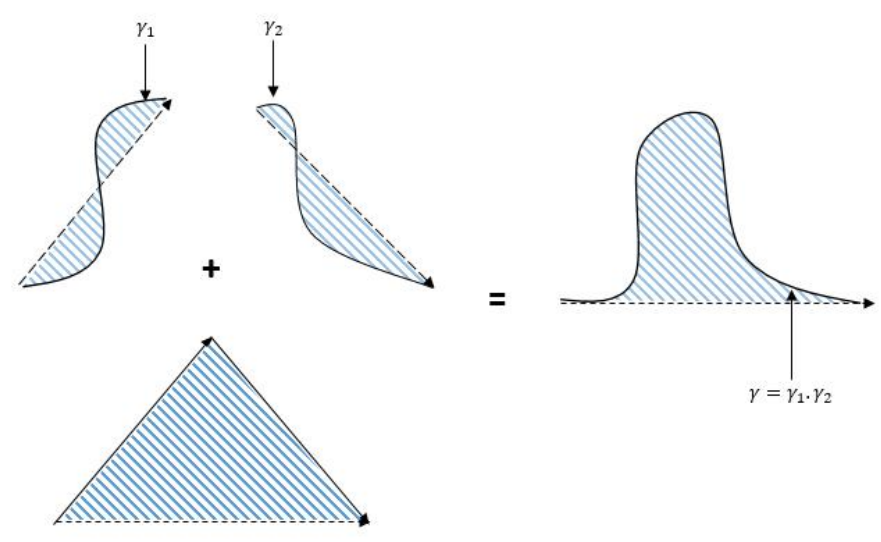

Figure 2.2. A geometric demonstration of Chen's rule on the area of concatenated paths.

Remark 2.4. In view of Proposition 2.3, one can use its assertion as a definition for $\alpha$-Hölder rough paths. This is sometimes preferable if one wishes to avoid the Lie group construction. However, in this paper we find the group presentation useful, mainly for the proof of the main result of the paper, Theorem 3.3, see section 6 . Moreover, Step 1 and 4 of the proof are based on Breuillard et al. (2009) which is formulated in the Lie group language. Also, the group actions are useful for presenting computations in a compact way, see in Step 2 of the proof. 


\section{Main result}

For a sequence $X=\left(X_{n}\right)_{n}$ of elements of $\mathbb{R}^{d}$, its continuous rescaled version $X^{(N)}$ is defined by

$$
X_{t}^{(N)}=\frac{1}{\sqrt{N}}\left(X_{\lfloor N t\rfloor}+(N t-\lfloor N t\rfloor)\left(X_{\lfloor N t\rfloor+1}-X_{\lfloor N t\rfloor}\right)\right) .
$$

We denote the lift of $X^{(N)}$ to a rough path by

$$
\iota^{(N)}(X)_{s, t}:=\left(X_{s, t}^{(N)}, S_{s, t}\left(X^{(N)}\right)\right),
$$

where $S_{s, t}\left(X^{(N)}\right)$ is the second level iterated integral of $X^{(N)}$ between $s$ and $t$, $S_{s, t}:=S_{s, t}^{2}$, as defined in (1.1), and the integration is in the Riemann-Stieltjes sense (which is well-defined since $X^{(N)}$ is of bounded variation on every compact interval $[s, t]$ ). One can check that for natural numbers $m<n$, the associated second level iterated integral has the following form

$$
S_{m, n}^{i, j}\left(X^{(1)}\right)=\sum_{m+1 \leq k<\ell \leq n} \Delta X_{k}^{i} \Delta X_{\ell}^{j}+\frac{1}{2} \sum_{m+1 \leq k \leq n} \Delta X_{k}^{i} \Delta X_{k}^{j},
$$

where $\Delta X_{k}:=X_{k}-X_{k-1}=X_{k-1, k}$ are the increments.

Definition 3.1. For a path $Y$ in $\mathbb{R}^{d}$ of bounded variation we define the area

$$
A_{s, t}^{i, j}(Y)=\frac{1}{2}\left(S_{s, t}^{i, j}(Y)-S_{s, t}^{j, i}(Y)\right)
$$

as the antisymmetric part of the iterated integral of $Y$. Set also $S_{t}(Y):=S_{0, t}(Y)$ and $A_{t}(Y):=A_{0, t}(Y)$.

Definition 3.2. Let $(X, \mathcal{F}, \mathbb{P})$ be a discrete time stochastic process on $\mathbb{R}^{d}$. We say that $X$ admits a regeneration structure if there are $\mathcal{F}$-measurable integer valued random variables $\left(\tau_{k}\right)_{k \in \mathbb{N}_{0}}$ so that $0=\tau_{0}<\tau_{1}<\tau_{2}<\ldots<\infty \mathbb{P}$-a.s. and

$$
\left(\tau_{k}-\tau_{k-1},\left\{X_{\tau_{k-1}, \tau_{k-1}+m}: 0 \leq m \leq \tau_{k}-\tau_{k-1}\right\}\right)
$$

are independent random variables for $k \geq 1$, and have the same distribution for all $k \geq 2$.

Theorem 3.3. Let $X$ be a discrete time stochastic process on $\mathbb{R}^{d}$ with bounded jumps $\left|X_{n+1}-X_{n}\right|_{\mathbb{R}^{d}} \leq K \mathbb{P}$-a.s. Assume that $X$ admits a regeneration structure in the sense of Definition 3.2 and let $\left(\tau_{k}\right)_{k \geq 0}$ be the corresponding regeneration times. Assume further that $X$ satisfies a strong law of large numbers

$$
\mathbb{P}\left(\lim _{n \rightarrow \infty} \frac{X_{n}}{n}=v\right)=1 .
$$

In this case the speed $v \in \mathbb{R}^{d}$ is defined by

$$
v:=\frac{\mathbb{E}\left[X_{\tau_{1}, \tau_{2}}\right]}{\mathbb{E}\left[\tau_{2}-\tau_{1}\right]}
$$

Also, assume that $\bar{X}_{n}=X_{n}-n v$ satisfies an annealed invariance principle with covariance matrix

$$
M=\frac{\mathbb{E}\left[\bar{X}_{\tau_{1}, \tau_{2}}^{(1)} \otimes \bar{X}_{\tau_{1}, \tau_{2}}^{(1)}\right]}{\mathbb{E}\left[\tau_{2}-\tau_{1}\right]}
$$


The last assumption is the following moment condition:

$$
\mathbb{E}\left[\left(\tau_{k}-\tau_{k-1}\right)^{2 p}\right]<\infty
$$

for some $p \geq 4$. Then we have the following weak convergence with respect to $\mathbb{P}$ to

$$
\iota^{(N)}(\bar{X}) \Rightarrow\left(B_{s, t}, \mathcal{S}_{s, t}+(t-s) \Gamma\right)_{0 \leq s<t \leq T} \text { in } \mathcal{C}^{\alpha}\left([0, T], G^{2}\left(\mathbb{R}^{d}\right)\right)
$$

for all $\alpha \in\left(\frac{1}{3}, \frac{p^{*}-1}{2 p^{*}}\right)$, where $p^{*}=\min \{\lfloor p\rfloor, 2\lfloor p / 2\rfloor\}$, and the couple $(B, \mathcal{S})$ are the Brownian motion with covariance matrix $M$ and its second level iterated Stratonovich integral process. Moreover, the correction is the antisymmetric matrix

$$
\Gamma=\frac{\mathbb{E}\left[A_{\tau_{1}, \tau_{2}}\left(\bar{X}^{(1)}\right)\right]}{\mathbb{E}\left[\tau_{2}-\tau_{1}\right]} .
$$

In particular, if the moment condition holds true for all $1 \leq p<\infty$ then the convergence holds true for all $\alpha<\frac{1}{2}$.

Remark 3.4. The corresponding result for the area with the same correction $\Gamma$ holds as well, that is whenever the path is considered with the antisymmetric operation, $S(X)$ is replaced by $A(X)$ and the enhanced Brownian motion $\mathcal{S}$ is replaced by the Stratonovich Levy area $\mathcal{A}$.

The correction matrix $\Gamma$ has the following decomposition.

Lemma 3.5. The following decomposition holds

$$
\begin{aligned}
\Gamma=\frac{\mathbb{E}\left[A_{\tau_{1}, \tau_{2}}\left(X^{(1)}\right)\right]}{\mathbb{E}\left[\tau_{2}-\tau_{1}\right]} \\
\quad+\frac{\mathbb{E}\left[\sum_{\tau_{1}<k<\ell \leq \tau_{2}}\left(v \otimes \Delta X_{k}+\Delta X_{\ell} \otimes v\right)-\left(\Delta X_{k} \otimes v+v \otimes \Delta X_{\ell}\right)\right]}{2 \mathbb{E}\left[\tau_{2}-\tau_{1}\right]} .
\end{aligned}
$$

Proof: One has

$$
\Delta \bar{X}_{\ell} \otimes \Delta \bar{X}_{k}=\Delta X_{\ell} \otimes \Delta X_{k}+v^{\otimes 2}-\left(v \otimes \Delta X_{k}+\Delta X_{\ell} \otimes v\right)
$$

since $\bar{X}_{n}=X_{n}-n v$. Neglecting the symmetric term we get the assertion

\section{Simple applications}

In this section we construct processes lacking or having non-zero area anomaly with a simple but instructive description. For starters, going back to the two processes compared in Chapter 2, the process with four steps clockwise loops every two steps (see Figure 2.1) have a non-zero area anomaly, while the process which stands still for four steps every two steps have no correction. For a discrete time process $X$, we remind the reader the notation $X^{n}(t):=\frac{X_{\lfloor n t\rfloor}}{\sqrt{n}}+\frac{1}{\sqrt{n}}\left((n t-\lfloor n t\rfloor)\left(X_{\lfloor n t\rfloor+1}-\right.\right.$ $\left.\left.X_{\lfloor n t\rfloor}\right)\right)$.

4.1. Rough path version of Donsker's Theorem (Breuillard et al., 2009; Kelly, 2016). Consider a discrete time random walk $X$ on $\mathbb{R}^{d}$. Assume that the increments $\Delta X_{n}$ are i.i.d. non-zero centered with finite $2 p$ moment for some $p \geq 4$. Then $\iota^{(N)}(X)_{\rightarrow}(B, \mathcal{S})$ in distribution in $\mathcal{C}^{\alpha}\left([0,1], G^{2}\left(\mathbb{R}^{2}\right)\right), \alpha \in\left(\frac{1}{3}, \frac{1}{2}-\frac{1}{p^{*}}\right)$, where $p^{*}=$ $\min (\lfloor p\rfloor, 2\lfloor p / 2\rfloor), \quad B$ is a $d$-dimensional Brownian motion, and $(\mathcal{S})$ is the Stratonovich second level iterated integral of $B$ in the time interval $[0,1]$. One 
can see this as a special case of our theorem for the case the regeneration times are the set of natural numbers.

Note that in this example no area correction appears. Since centering a random walk with a drift defines a new random walk with no drift, this example shows that a non-zero area anomaly cannot be created only from the presence of drift.

4.2. Rotating drift (Lopusanschi and Simon, 2018). The following example shows that non-zero area anomaly is possible even with no speed. Consider $\mathbb{Z}^{2} \subset \mathbb{C}$. Let $\left(\zeta_{n}\right)_{n}$ be i.i.d. so that $P\left(\zeta_{1}=1\right)=p=1-P\left(\zeta_{1}=-1\right)$. Define $\Delta X_{n}:=i^{n} \zeta_{n}$, $i=\sqrt{-1}$. Then $X^{N} \rightarrow B$ in distribution in the uniform topology, $B$ is a BM with covariance $2 p(1-p) \mathcal{I}$, where $\mathcal{I}$ is the identity matrix. However, after rescaling $\iota^{(N)}(X) \rightarrow(B, \mathcal{S}+\Gamma \cdot)$ in distribution in $\mathcal{C}^{\alpha}\left([0,1], G^{2}\left(\mathbb{R}^{2}\right)\right), \alpha<1 / 2$, where $\mathcal{S}$ is the Stratonovich second level iterated integral of $B$ in $[0,1]$.

Indeed, $X$ has a regeneration structure for the deterministic times $\tau_{k}:=4 k, k \geq$ 0 , which, trivially, have all moments. One can check that there is a strong law of large numbers with speed $v=0$. Then $\Gamma=\frac{1}{4} \mathbb{E}\left[A_{0,4}(X)\right]$, and straight forward computation yields

$$
\Gamma=\frac{(2 p-1)^{2}}{4}\left(\begin{array}{cc}
0 & 1 \\
-1 & 0
\end{array}\right)
$$

which is non-zero if $p \neq \frac{1}{2}$.

Different presentation: non-elliptic periodic environment. The same example as above can be presented as a walk in a certain fixed space non-homogenous environment rather than a walk with drift rotating in time. Consider again $\mathbb{Z}^{2}$ as a subset of $\mathbb{C}$ and fix some $0<p<1$. Let $\omega$ be the two-periodic environment given by: $\omega(0,1)=p=1-\omega(0,-1), \omega(1,1+i)=p=1-\omega(1,1-i)$, $\omega(1+i, i)=p=1-\omega(1+i, 2+i)$, and $\omega(i, 0)=p=1-\omega(i, 2 i)$. In particular, twoperiodicity means that $\omega(v, w)=\omega(v+z, w)=\omega(v, w+z)$ for every $w, v \in \mathbb{Z}+\mathbb{Z} i$ and $z \in 2 \mathbb{Z}+2 \mathbb{Z} i$. Finally, let $X$ be the Markov chain on $\mathbb{Z}^{2}$ with transition probabilities $\omega$. Then, by parity, the law of $X$ is the same as the law of the last rotating drift example. In particular, the same result holds for this example as well.

\section{Applications}

5.1. Random walks in random environment. We first define random walks in random environment on $\mathbb{Z}^{d}$. Let $\mathcal{E}:=\left\{e_{i}: i=1, \ldots, 2 d\right\} \subset \mathbb{Z}^{d}$ be the set of neighbors of the origin. Let $\mathcal{P}_{x}$ be the space of probability distributions on the algebraic sum $x+\mathcal{E}:=\{x+e: e \in \mathcal{E}\}$. We call $\Omega=\prod_{x \in \mathbb{Z}^{d}} \mathcal{P}_{x}$ the space of environments on $\mathbb{Z}^{d}$. In particular, an environment $\omega \in \Omega$ is of the form $\omega=(\omega(x, x+e))_{x \in \mathbb{Z}^{d}, e \in \mathcal{E}}$ so that $\omega(\cdot, \cdot) \geq 0$ and $\sum_{e \in \mathcal{E}} \omega(\cdot, \cdot+e)=1$.

For a fixed environment $\omega \in \Omega$ and a starting point $x \in \mathbb{Z}^{d}$ we define a nearest neighbor walk $X$ on $\mathbb{Z}^{d}$ to be the Markov chain starting at $x, P_{x, \omega}\left(X_{0}=x\right)=1$, with transition probabilities $P_{x, \omega}\left(X_{n+1}=y+e \mid X_{n}=y\right)=\omega(y, y+e)$. Given a probability distribution $P$ on $\Omega$, the annealed (and sometimes called also the averaged) law of the walk $X$ is characterized by $\mathbb{P}_{x}(\cdot):=\int P_{x, \omega}(\cdot) \mathrm{d} P(\omega)$. We also call $P_{x, \omega}$ the quenched law. We say that the environment is i.i.d. if $\left(\omega_{x}\right)_{x \in \mathbb{Z}^{d}}$ is an i.i.d. sequence under $P$. An i.i.d. random environment is called uniformly elliptic if there is some deterministic $\kappa>0$ so that $P(\omega(0, e) \geq \kappa$ for all $e \in \mathcal{E})=1$. 
We now define some ballisticity conditions and for that adapt the notation of Berger et al. (2014). Fix $L \geq 0$ and let $\ell \in \mathbb{S}^{d-1}$ be an element of the unit sphere. Then we write

$$
H_{L}^{\ell}:=\inf \left\{n \in \mathbb{N}_{0}: X_{n} \cdot \ell>L\right\}
$$

for the first entrance time of $\left(X_{n}\right)$ into the half-space $\left\{x \in \mathbb{Z}^{d}: x \cdot \ell>L\right\}$, where $\mathbb{N}_{0}=\{0,1,2, \ldots\}$.

Definition 5.1 (Sznitman $\left(T^{\prime}\right) \mid \ell$ condition, Sznitman, 2002). Let $\gamma \in(0,1]$ and $l \in \mathbb{S}^{d-1}$. We say that condition $(T)_{\gamma}$ is satisfied with respect to $\ell$, and write $(T)_{\gamma} \mid \ell$, if for every $b>0$ and each $\ell^{\prime}$ in some neighborhood of $\ell$ one has that

$$
\limsup _{L \rightarrow \infty} L^{-\gamma} \ln \mathbb{P}_{0}\left(H_{L}^{\ell^{\prime}}>H_{b L}^{-\ell^{\prime}}\right)<0 .
$$

We say that condition $\left(T^{\prime}\right)$ is satisfied with respect to $\ell$, and write $\left(T^{\prime}\right) \mid \ell$, if condition $(T)_{\gamma} \mid \ell$ is fulfilled for every $\gamma \in(0,1)$.

Definition 5.2 (Berger-Drewitz-Ramirez $\left(P_{M}^{*} \mid \ell\right)$ condition, Berger et al., 2014). Let $M>0$ and $\ell \in \mathbb{S}^{d-1}$. We say that condition $P_{M}^{*} \mid \ell$ is satisfied with respect to $\ell$ if for every $b>0$ and all $\ell^{\prime} \in \mathbb{S}^{d-1}$ in some neighborhood of $\ell$, one has that

$$
\limsup _{L \rightarrow \infty} L^{M} P_{0}\left(H_{b L}^{-\ell^{\prime}}<H_{L}^{\ell^{\prime}}\right)=0
$$

Theorem 5.3. Let $X$ be a random walk in i.i.d. and uniformly elliptic random environment on $\mathbb{Z}^{d}$, where $d \geq 2$. Let $\ell \in \mathbb{S}^{d-1}$ and assume that the Sznitman-type condition $P_{M}^{*} \mid \ell$ of Berger-Drewitz-Ramirez holds for some $M>15 d+5$. Then, considering $X$ with respect to the annealed law $\mathbb{P}_{0}$, the conditions of Theorem 3.3 are satisfied, and moreover the moment condition holds for all $1 \leq p<\infty$.

Proof: Berger, Drewitz, and Ramirez (Berger et al., 2014, Theorem 1.6) states that in this case the stronger condition $\left(T^{\prime}\right) \mid \ell$ of Sznitman also holds. The law of large numbers, including the existence of regeneration times were proved in Sznitman and Zerner (1999) where the independence mentioned only the increments $\left(X_{\tau_{k-1}, \tau_{k}}, \tau_{k}-\tau_{k-1}\right)$. However, the proof of Sznitman and Zerner (1999) shows that the walk on different intervals $\left(X_{\tau_{k-1}, \tau_{k-1}+m} \cdot \ell\right)_{m \leq \tau_{k}-\tau_{k-1}}$, is independent for $k \geq 1$ and identically distributed for $k \geq 2$, and, moreover, the walk satisfies $X_{\tau_{k-1}, \tau_{k-1}+m} \cdot \ell>0$ for all $m>0$. This form appears specifically, e.g., in Berger (2008, Claim 3.4). In particular, $X$ admits a regeneration structure in the sense of Definition 3.2. Annealed invariance principle was proved in Sznitman (2000, Theorem 4.1) and Sznitman (2001, Theorem 3.6) based on the finiteness of all moments for the regeneration time, which was proved in Sznitman (2001, Theorem 3.4).

Remark 5.4. A version of a well-known conjecture by Sznitman is as follows: For random walks in random environment on $\mathbb{Z}^{d}, d \geq 2$, in i.i.d. and uniformly elliptic environment directional transience in some direction $\ell$ is enough for attaining finiteness of all moments for the regeneration times. Therefore, assuming the conjecture then directional transience in some direction $\ell$ is enough for an annealed convergence in the $\alpha$-Hölder rough path topology for all $\alpha<1 / 2$. In particular, one would not expect an example with a more singular convergence, or, more accurately no example for directionally transient i.i.d uniformly elliptic RWRE for which there are some $\alpha<\beta<1 / 2$ so that the convergence holds in $\alpha$-Hölder but not in $\beta$-Hölder. 
A Dirichlet distribution with parameters $\alpha_{1}>0, \ldots, \alpha_{N}>0$ is defined by the density $\varphi$ with respect to Lebesgue measure on $S^{N-1}:=\left\{x \in \mathbb{R}^{N}: x_{i} \geq 0, \sum_{i=1}^{N} x_{i}=\right.$ $1\}$, the $(N-1)$-dimensional simplex, defined by

$$
\varphi(x)=\frac{1}{B\left(\alpha_{1}, \ldots, \alpha_{N}\right)} \prod_{i=1}^{N} x^{\alpha_{1}-1},
$$

where $B\left(\alpha_{1}, \ldots, \alpha_{N}\right)$ is a normalizing constant. Let $X$ be a random walk in i.i.d. random environment so that $\omega_{0}$ has the Dirichlet distribution with parameters $\alpha_{e}>0$ for $e \in \mathcal{E}$. It is known that in dimension $d \geq 2$ if

$$
\sum_{e \in \mathcal{E}}\left|\alpha_{e}-\alpha_{-e}\right|>1
$$

then for every direction $\ell$ for which $\sum_{|e|=1} \alpha_{e} e \cdot \ell>0$ the walk can be decomposed into regeneration intervals in direction $\ell$ in the form that appears in the proof of Theorem 5.3 above, and in particular it admits a regeneration structure in the sense of Definition 3.2. Moreover, the regeneration interval $\tau_{2}-\tau_{1}$ has a finite $p$-th moment if and only if $p<\kappa$, where $\kappa$ is defined by

$$
\kappa=2 \sum_{e \in \mathcal{E}} \alpha_{e}-\max _{e \in \mathcal{E}}\left\{\alpha_{e}+\alpha_{-e}\right\}
$$

see Sabot and Tournier (2017, Corollary 2). In particular, we have

Theorem 5.5. Let $X$ be a random walk in i.i.d. Dirichlet environment $\mathbb{Z}^{d}$, where $d \geq 2$. Assume that (5.3) holds. If $\kappa>8$, then under the annealed law the conditions of Theorem 3.3 are satisfied with $p<\kappa / 2$. In particular, we have a convergence in the $\alpha$-Hölder rough path topology for all $\alpha \in\left(\frac{1}{3}, \frac{1}{2}-\frac{1}{(\kappa / 2)^{*}}\right)$, where $(\kappa / 2)^{*}=\min \{\lfloor\kappa / 2\rfloor, 2\lfloor\kappa / 4\rfloor\}$.

Remark 5.6. It is relevant to point out here that the $\alpha$-Hölder rough topology is not the only choice one can make (although it is certainly more common). We chose to work with it in this paper due to availability of the results of Breuillard et al. (2009) and Kelly (2016) which were considered in these settings. However, without going into the details here, let us mention that one can also define a rough path topology using the $p$-variation norm, which is parameterization-free and corresponds to $1 / p$ Hölder topology. This was in fact the original definition in Lyons (1998). Using some recent available estimates, we believe that one should be able to prove a version of our Theorem 3.3 in the $p$-variation rough path topology, for every $p>2$, assuming only finiteness of the second moment of the jumps. The last example shows why this might be desirable. On the other hand, in the view of Remark 5.4, there's no advantage for $p$-variation rough paths if one is interested in RWRE from the ballistic class.

We close this section with an open problem. As one can notice in the examples given in Chapter 4, to construct a law with non-zero area anomaly it is not enough to have an asymptotic direction or non-trivial covariances. Area anomaly might hint that there is some asymmetry in the shape of the path with respect to the asymptotic direction. We conjecture that, roughly speaking, any "reasonably asymmetric" RWRE from the ballistic class considered in Theorem 5.3 would have a non-zero area anomaly. However, the following is still an open problem. 
Problem 5.7. Is there a RWRE satisfying the conditions of Theorem 5.3 for which the area anomaly $\Gamma$ is non-zero? Note that the question is open even for stationary and ergodic RWRE.

5.2. Periodic graphs or hidden Markov walks. Theorem 3.3 naturally generalizes the main results in Lopusanschi and Simon (2018) and Lopusanschi and Simon (2017).

Theorem 5.8 (Lopusanschi and Simon, 2017, 2018). Let X be either an irreducible Markov chain on a periodic graph (see the definition in Lopusanschi and Simon, 2018) or an irreducible hidden Markov walk driven by a finite state Markov chain (see the definition in Lopusanschi and Simon, 2017), then the conditions of Theorem 3.3 are satisfied.

Proof (Sketch): If $\left(Y_{n}\right)_{n}$ is an irreducible Markov chain on a periodic graph or an irreducible hidden Markov walk, it admit an underlying irreducible Markov chain $\left(X_{n}\right)_{n}$ on a finite state space. More precisely, for every $n \geq 1$, the increment $Y_{n+1}-Y_{n}$ depends on $X_{n}$ in an appropriate way.

We can thus define a sequence on stopping times for $\left(X_{n}\right)_{n}$ as

$$
T_{0}=0 \text { and } T_{n}=\inf \left\{k>T_{n-1}: X_{k}=X_{0}\right\}, n \geq 1 .
$$

In particular, it is a sequence of return times to the initial position of $\left(X_{n}\right)_{n \geq 0}$. By construction, the sequence $\left(T_{n}\right)_{n>0}$ is strictly increasing and, as $\left(X_{n}\right)_{n>0}$ is irreducible, all $T_{n}$ are finite a.s. The increments $\left(T_{n+1}-T_{n}\right)_{n \geq 0}$ are i.i.d., as well as the variables $\left(Y_{T_{n+1}}-Y_{T_{n}}\right)_{n \geq 0}$ (see the proof in Lopusanschi and Simon, 2018) and, more generally,

$$
\left(\left(Y_{T_{n}+m}-Y_{T_{n}}\right)_{0 \leq m \leq T_{n+1}-T_{n}}, T_{n+1}-T_{n}\right)_{n \geq 0} .
$$

Consequently the process $\left(Y_{n}\right)_{n \geq 0}$ admits a regeneration structure.

Moreover, since $\left(X_{n}\right)_{n \geq 0}$ is irreducible and takes values on a finite state space, all moments of the increments $T_{n+1}-T_{n}$ are finite (they actually have geometric tails). Concluding the law of large numbers and the invariance principle is now routine.

\section{Proof of Theorem 3.3}

We shall take the general route of Lopusanschi and Simon (2017), where the authors proved first the convergence for the path on a sequence of return times with exponential tails, and then moved to the full path, where they identified an

area correction. For both identification of the limit and tightness they used the strong Markov property together with the the tail bounds of the stopping times. To demonstrate the idea in a rather simple way the reader is suggested to think about the case of random walks on a deterministic periodic environment on $\mathbb{Z}^{d}$, where the decomposition is done according to return times of the walk to the origin modulu the period. In our proof, we decompose the path according to the regeneration times, which are not stopping times and therefore the strong Markov property does not apply. However, as we shall show, the i.i.d. nature of our decomposition together with the finiteness of the regeneration time interval moments are enough to conclude.

Proof of Theorem 3.3: The proof will be divided in four steps: 
- Convergence in distribution of the centered discrete process given by the sum of $\bar{X}_{\tau_{k}, \tau_{k+1}}$ using the rough path version of Donsker's Theorem.

- Convergence of the finite-dimensional marginals of the subsequence $\left(\iota^{\left(\tau_{k}\right)}(\bar{X})\right)_{k \geq 1}$, where we see the area anomaly $\Gamma$.

- Convergence of finite-dimensional marginals of the full process $\left(\iota^{(N)}(\bar{X})\right)_{N \geq 1}$.

- Tightness of the sequence $\left(\iota^{(N)}(\bar{X})\right)_{N \geq 1}$.

Step 1: Let $Y_{n}=: \bar{X}_{\tau_{n}}$. We claim that $\iota^{(N)}(Y)_{t<T} \rightarrow\left(B_{t}^{\prime}, \mathcal{S}_{t}^{\prime}\right)_{t<T}$ in distribution with respect to $\mathbb{P}_{0}$ in $\mathcal{C}^{\alpha}\left([0, T], G^{2}\left(\mathbb{R}^{d}\right)\right)$ for all $\alpha \in\left(\frac{1}{3}, \frac{1}{2}-\frac{1}{2 p^{*}}\right)$, where $B^{\prime}$ is a Brownian motion with covariance matrix $\mathbb{E}\left[\bar{X}_{\tau_{1}, \tau_{2}} \otimes \bar{X}_{\tau_{1}, \tau_{2}}\right]$ and $\mathcal{S}^{\prime}$ is its corresponding second level iterated Stratonovich integral. Indeed, assuming without loss of generality that $\left(\bar{X}_{\tau_{1}}, \tau_{1}\right)$ has the same distribution of $\left(\bar{X}_{\tau_{1}, \tau_{2}}, \tau_{2}-\tau_{1}\right)$, then $Y_{n}=\sum_{i=1}^{n} \Delta Y_{i}$ is a sum of i.i.d. centered random variables with values in $\mathbb{R}^{d}$ and with covariance $\mathbb{E}\left[\Delta Y_{1} \otimes \Delta Y_{1}\right]=\mathbb{E}\left[\bar{X}_{\tau_{1}, \tau_{2}} \otimes \bar{X}_{\tau_{1}, \tau_{2}}\right]$. Moreover, since the jumps are $\mathbb{P}_{0}$-a.s. bounded $\left|\Delta X_{n}\right|_{\mathbb{R}^{d}} \leq K$, then $\left|\Delta Y_{n}\right|_{\mathbb{R}^{d}} \leq R\left(\tau_{n}-\tau_{n-1}\right)$ and therefore also have finite $2 p$ moment, where $R=R(K, d)$ is some constant. Applying Theorem 1 of Breuillard et al. (2009) to the process $D^{-1 / 2} Y_{n}$, where $D=\mathbb{E}\left[\bar{X}_{\tau_{1}, \tau_{2}} \otimes \bar{X}_{\tau_{1}, \tau_{2}}\right]$, we get weak convergence of $Y^{(N)}$ in in $\mathcal{C}^{\alpha}\left([0, T], G^{2}\left(\mathbb{R}^{d}\right)\right)$ for all $\alpha \in\left(\frac{1}{3}, \frac{1}{2}-\frac{1}{2 p^{*}}\right)$. (Alternatively, Lemma 3.1 of Kelly (2016) with $V=1$ in the equation appearing there implies the convergence in uniform topology and therefore the convergence of the finite-dimensional marginals, then the tightness in $\mathcal{C}^{\alpha}\left([0, T], G^{2}\left(\mathbb{R}^{d}\right)\right)$ for all $\alpha \in\left(\frac{1}{3}, \frac{1}{2}-\frac{1}{2 p^{*}}\right)$ is showed in the proof of that lemma using the Kolmogorov Criterion.)

Step 2: Denote by $\delta_{\epsilon}$ the standard dilatation by $\epsilon$, that is $\delta_{\epsilon}(x, a)=\left(\epsilon x, \epsilon^{2} a\right)$. By (2.1) and (3.2) we have the following decomposition of the rough path lift of $\bar{X}$

$$
\delta_{N^{1 / 2} \iota}^{(N)}(\bar{X})_{\frac{m}{N}}=\bigotimes_{k=1}^{m}\left(\Delta \bar{X}_{k}, \frac{1}{2} \Delta \bar{X}_{k}^{\otimes 2}\right)
$$

Then, using the properties of integrals for piecewise linear processes, for $r \in \mathbb{N}$, we get the decomposition

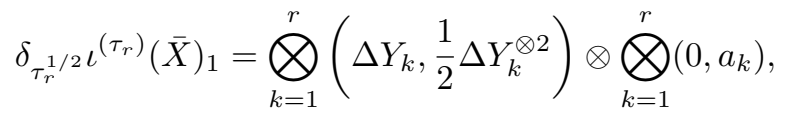

where

$$
a_{k}=\frac{1}{2} \sum_{\tau_{k-1}+1 \leq m<n \leq \tau_{k}}\left(\Delta \bar{X}_{n} \otimes \Delta \bar{X}_{m}-\Delta \bar{X}_{m} \otimes \Delta \bar{X}_{n}\right)
$$

is the discrete area between the times $\tau_{k-1}$ and $\tau_{k}$. We note that the first term in the product at the right hand side of (6.1) corresponds to the rough path of a partial sum of our i.i.d. variables $\Delta\left(\bar{X}_{\tau}\right)_{k}$. We have seen in step 1 that the sequence of rough paths which is corresponding to these partial sums converges in distribution to the enhanced Brownian motion in the $\alpha$-Hölder topology, which implies that the corresponding finite-dimensional marginals converge in distribution to those of the Brownian motion.

On the other hand, for every fixed $s \in \mathbb{N}$ and $0<t_{1}<\ldots<t_{s}$, using the fact that the process $X$ admits a regeneration structure, we conclude that $a_{k}, k \geq 2$, are i.i.d., and moreover each coordinate of $a_{k}$ is bounded by a multiple of $K\left(\tau_{k}-\tau_{k-1}\right)^{2}$, 
which has a bounded expectation. Thus, by the law of large numbers, we have the following convergence

$$
\left(\frac{1}{r} \sum_{k=1}^{r} a_{\left\lfloor t_{1} k\right\rfloor}, \ldots, \frac{1}{r} \sum_{k=1}^{r} a_{\left\lfloor t_{s} k\right\rfloor}\right) \underset{r \rightarrow \infty}{\longrightarrow} \mathbb{E}\left[a_{2}\right]\left(t_{1}, \ldots, t_{s}\right) \text { a.s. }
$$

Moreover, the law of large numbers implies $\frac{\tau_{k}}{k} \rightarrow \mathbb{E}\left[\tau_{2}-\tau_{1}\right]=: \beta \mathbb{P}_{0}$-a.s. Since $\bigotimes_{k=1}^{r}\left(0, a_{k}\right)=\left(0, \sum_{k=1}^{r} a_{k}\right)$, we can use Slutsky's theorem (Slutsky, 1925) as in Lopusanschi and Simon (2017, Lemma 2.3.2) to conclude that we have the following convergence in distribution

$$
\left(\iota^{\left(\tau_{r}\right)}(\bar{X})_{t_{1}}, \ldots, \iota^{\left(\tau_{r}\right)}(\bar{X})_{t_{s}}\right) \underset{r \rightarrow \infty}{\longrightarrow}\left(\left(B_{t_{1}}, \mathcal{S}_{t_{1}}+t_{1} \Gamma\right), \ldots,\left(B_{t_{s}}, \mathcal{S}_{t_{s}}+t_{s} \Gamma\right)\right)
$$

where $\Gamma=\beta^{-1} \mathbb{E}\left[a_{2}\right]$ is an antisymmetric matrix, $B=\beta^{-1 / 2} B^{\prime}$ and $\mathcal{S}$ is its corresponding Stratonovich iterated integral.

Step 3: Set $\kappa(n)$ to be the unique integer such that $\tau_{\kappa(n)} \leq n<\tau_{\kappa(n)+1}$. We use (2.3) together with the fact that $\bar{X}$ has bounded increments a.s. to deduce

$$
\begin{aligned}
& \mathbf{d}\left(\delta_{N^{-1 / 2}}\left(\iota^{(1)}(X)_{\tau_{\kappa(\lfloor N t\rfloor)}}\right), \delta_{N^{-1 / 2}}\left(\iota^{(1)}(X)_{\lfloor N t\rfloor}\right)\right) \\
& =N^{-1 / 2} \mathbf{d}\left(\left(\iota^{(1)}(X)_{\tau_{\kappa(\lfloor N t\rfloor)}}\right),\left(\iota^{(1)}(X)_{\lfloor N t\rfloor}\right)\right) \\
& \leq d K N^{-1 / 2}\left(\lfloor N t\rfloor-\tau_{\kappa(\lfloor N t\rfloor)}\right) .
\end{aligned}
$$

Applying the Markov inequality we obtain the following convergence for any $\epsilon>0$

$$
\begin{aligned}
& \mathbb{P}\left(\mathbf{d}\left(\delta_{N^{-1 / 2}}\left(\iota^{(1)}(X)_{T_{\kappa(\lfloor N t\rfloor)}}\right), \delta_{N^{-1 / 2}}\left(\iota^{(1)}(X)_{\lfloor N t\rfloor}\right)\right)>\epsilon\right) \\
& \leq K d \frac{\mathbb{E}\left[\lfloor N t\rfloor-\tau_{\kappa(\lfloor N t\rfloor)}\right]}{N^{1 / 2} \epsilon} \\
& \leq K d \frac{\mathbb{E}\left[\tau_{\kappa(\lfloor N t\rfloor)+1}-\tau_{\kappa(\lfloor N t\rfloor)}\right]}{N^{1 / 2} \epsilon} .
\end{aligned}
$$

Note that $\mathbb{E}\left[\tau_{\kappa(\lfloor N t\rfloor)+1}-\tau_{\kappa(\lfloor N t\rfloor)}\right] \leq\left(\mathbb{E}\left[\tau_{2}^{3}\right] t\right)^{1 / 3} N^{1 / 3}$ as $\tau_{2}$ has a finite third moment. Indeed,

$$
\begin{aligned}
\mathbb{E}\left[\tau_{\kappa(\lfloor N t\rfloor)+1}-\tau_{\kappa(\lfloor N t\rfloor)}\right] & =\sum_{k=0}^{N t} \mathbb{E}\left[\left(\tau_{k+1}-\tau_{k}\right) \mathbb{1}_{\kappa(\lfloor N t\rfloor)=k}\right] \\
& \leq \sum_{k=0}^{N t} \mathbb{E}\left[\left(\tau_{k+1}-\tau_{k}\right)^{3}\right]^{1 / 3} \mathbb{P}(\kappa(\lfloor N t\rfloor)=k)^{2 / 3} \\
& \leq \mathbb{E}\left[\tau_{2}^{3}\right]^{1 / 3} \sum_{k=0}^{N t} \mathbb{P}(\kappa(\lfloor N t\rfloor)=k)^{2 / 3} \\
& \leq \mathbb{E}\left[\tau_{2}^{3}\right]^{1 / 3}(N t)^{1 / 3}
\end{aligned}
$$

Therefore,

$$
\mathbb{P}\left(\mathbf{d}\left(\delta_{N^{-1 / 2}}\left(\iota^{(1)}(X)_{\tau_{\kappa(\lfloor N t\rfloor)}}\right), \delta_{N^{-1 / 2}}\left(\iota^{(1)}(X)_{\lfloor N t\rfloor}\right)\right)>\epsilon\right) \leq \frac{K d\left(\mathbb{E}\left[\tau_{2}^{3}\right] t\right)^{1 / 3}}{N^{1 / 6} \epsilon} \rightarrow 0
$$

as $N \rightarrow \infty$. Next, using the strong law of large numbers together with the decomposition of $\tau_{k}=\sum_{\ell=1}^{k}\left(\tau_{\ell}-\tau_{\ell-1}\right)$ into independent variables, with the same distribution for $\ell>1$, one deduces that $\kappa(n) / n$ converges a.s. to $\beta^{-1}$. Hence the 
conclusion of Step 2 together with Slutsky's Theorem (Slutsky, 1925) imply the convergence in distribution

$$
\iota^{(N)}(X) \rightarrow\left(B_{t}, \mathcal{S}_{t}+t \Gamma\right)
$$

for any fixed $t \in[0, T]$. Extending the convergence to all finite-dimensional marginals of $\iota^{(N)}(X)$ is done similarly using Slutsky's Theorem on $\mathbb{R}^{d} \times \mathbb{R}^{d \otimes d}$.

Step 4: It is left to prove the tightness of the process. In order to do this, we use the Kolmogorov tightness criterion for rough paths Friz and Hairer (2014, Theorem 3.10). That is, in order to obtain tightness for $\alpha<\frac{p^{*}-1}{2 p^{*}}$. it is enough to show that there exists a positive constant $c$ such that, for all $0 \leq s<t \leq T$,

$$
\sup _{N} \mathbb{E}\left[\left\|\iota^{(N)}(X)_{s, t}\right\|_{G^{2}\left(\mathbb{R}^{d}\right)}^{2 p^{*}}\right] \leq c|t-s|^{p^{*}} .
$$

To avoid heavy notation we write $\mathbf{X}_{s, t}:=\iota^{(1)}(X)_{s, t}$ and assume without loss of generality that $\tau_{1}$ has the same distribution as $\tau_{k}-\tau_{k-1}$ for $k>1$. From the definition of iterated integral and the fact the paths are linear interpolations of discrete paths proving (6.2) boils down to showing that there is a constant $c$ so that

$$
\mathbb{E}\left[\left\|\mathbf{X}_{\ell, k}\right\|_{G^{2}\left(\mathbb{R}^{d}\right)}^{2 p^{*}}\right] \leq c(k-\ell)^{p^{*}}
$$

uniformly on $0 \leq \ell<k \leq N T$. Note that by the i.i.d regeneration structure

$$
\mathbb{E}\left[\left\|\mathbf{X}_{\tau_{\ell}, \tau_{k}}\right\|_{G^{2}\left(\mathbb{R}^{d}\right)}^{2 p^{*}}\right]=\mathbb{E}\left[\left\|\mathbf{X}_{\tau_{k}-\tau_{\ell}}\right\|_{G^{2}\left(\mathbb{R}^{d}\right)}^{2 p^{*}}\right] .
$$

The tightness argument Breuillard et al. (2009, Step 2 in Chapter 3) then immediately implies

$$
\mathbb{E}\left[\left\|\mathbf{X}_{\tau_{k}}\right\|_{G^{2}\left(\mathbb{R}^{d}\right)}^{2 p^{*}}\right]=O\left(k^{p^{*}}\right)
$$

where we used the fact that $\mathbb{E}\left[\tau_{k}^{p^{*}}\right]=O\left(k^{p^{*}}\right)$. Next, if $k, \ell$ are in the same regeneration interval, the fact that the jumps are bounded, regeneration intervals have finite $2 p^{*}$ moments, and the definition (2.3) imply

$$
\mathbb{E}\left[\left\|\mathbf{X}_{\ell, k}\right\|_{G^{2}\left(\mathbb{R}^{d}\right)}^{2 p^{*}} \mathbb{1}_{\kappa(\ell)=\kappa(k)}\right] \leq C_{p}^{\prime}
$$

for some constant $C_{p}^{\prime}$. Therefore by sub-additivity (2.4), and using Hölder's inequality together with (2.5) we can find a constant $C_{2 p^{*}}$ so that

$$
\mathbb{E}\left[\left\|\mathbf{X}_{\ell, k}\right\|_{G^{2}\left(\mathbb{R}^{d}\right)}^{2 p^{*}}\right] \leq C_{2 p^{*}}\left(2 C_{p}^{\prime}+\mathbb{E}\left[\left\|\mathbf{X}_{\tau_{\kappa(\ell)}, \tau_{\kappa(k)+1}}\right\|_{G^{2}\left(\mathbb{R}^{d}\right)}^{2 p^{*}}\right]\right)=O\left((k-\ell)^{p^{*}}\right) .
$$

We conclude that the Kolmogorov criterion is satisfied and so the sequence $\left(\iota^{(N)}(X)\right)_{N}$ is tight in $\mathcal{C}^{\alpha}\left([0, T], G^{2}\left(\mathbb{R}^{d}\right), \alpha<\frac{p^{*}-1}{2 p^{*}}\right.$.

Remark 6.1. After the submission of an earlier version of this paper a variation of Theorem 3.3 was proved in Orenshtein (2021), where the rough path space is defined there with respect to the $p$-variation norm rather than the $\alpha$-Hölder norm used in this paper. The conditions on the jumps of the process were generalized there, and, in particular, for the case of bounded jumps assumed in Theorem 3.3 the moment condition in (3.4) was weakened to the case $p=1$, which is optimal. Another recent related result is a rough path version of Kipnis-Varadhan's Theorem for additive functionals of Markov processes (Deuschel et al., 2021). One application 
is to a different class of random walks in random media which have no regenerative structure - random walks among random conductances.

\section{Acknowledgements}

The authors would like to thank Damien Simon for a stimulating email exchange which contributed to an earlier version of the paper. They are also grateful to the anonymous referees for their useful comments.

\section{References}

Berger, N. Limiting velocity of high-dimensional random walk in random environment. Ann. Probab., 36 (2), 728-738 (2008). MR2393995.

Berger, N., Drewitz, A., and Ramírez, A. F. Effective polynomial ballisticity conditions for random walk in random environment. Comm. Pure Appl. Math., 67 (12), 1947-1973 (2014). MR3272364.

Breuillard, E., Friz, P., and Huesmann, M. From random walks to rough paths. Proc. Amer. Math. Soc., 137 (10), 3487-3496 (2009). MR2515418.

Bruned, Y., Hairer, M., and Zambotti, L. Algebraic renormalisation of regularity structures. Invent. Math., 215 (3), 1039-1156 (2019). MR3935036.

Chevyrev, I. and Friz, P. K. Canonical RDEs and general semimartingales as rough paths. Ann. Probab., 47 (1), 420-463 (2019). MR3909973.

Deuschel, J.-D., Orenshtein, T., and Perkowski, N. Additive functionals as rough paths. The Annals of Probability, 49 (3), 1450-1479 (2021). DOI: 10.1214/20AOP1488.

Friz, P., Gassiat, P., and Lyons, T. Physical Brownian motion in a magnetic field as a rough path. Trans. Amer. Math. Soc., 367 (11), 7939-7955 (2015). MR3391905.

Friz, P. K. and Hairer, M. A course on rough paths. With an introduction to regularity structures. Universitext. Springer, Cham (2014). ISBN 978-3-31908331-5; 978-3-319-08332-2. MR3289027.

Friz, P. K. and Victoir, N. B. Multidimensional stochastic processes as rough paths. Theory and applications, volume 120 of Cambridge Studies in Advanced Mathematics. Cambridge University Press, Cambridge (2010). ISBN 978-0-521-87607-0. MR2604669.

Friz, P. K. and Zhang, H. Differential equations driven by rough paths with jumps. J. Differential Equations, 264 (10), 6226-6301 (2018). MR3770049.

Gubinelli, M. Controlling rough paths. J. Funct. Anal., 216 (1), 86-140 (2004). MR2091358.

Gubinelli, M. Ramification of rough paths. J. Differential Equations, 248 (4), 693-721 (2010). MR2578445.

Hairer, M. A theory of regularity structures. Invent. Math., 198 (2), 269-504 (2014). MR3274562.

Ishiwata, S., Kawabi, H., and Namba, R. Central limit theorems for non-symmetric random walks on nilpotent covering graphs: Part I. Electron. J. Probab., 25, Paper No. 86, 46 (2020a). MR4125791.

Ishiwata, S., Kawabi, H., and Namba, R. Central Limit Theorems for NonSymmetric Random Walks on Nilpotent Covering Graphs: Part II. Potential Analysis (2020b). DOI: 10.1007/s11118-020-09851-7. 
Kelly, D. Rough path recursions and diffusion approximations. Ann. Appl. Probab., 26 (1), 425-461 (2016). MR3449323.

Kelly, D. and Melbourne, I. Smooth approximation of stochastic differential equations. Ann. Probab., 44 (1), 479-520 (2016). MR3456344.

Lejay, A. An introduction to rough paths. In Séminaire de Probabilités XXXVII, volume 1832 of Lecture Notes in Math., pp. 1-59. Springer, Berlin (2003). MR2053040.

Lopusanschi, O. and Simon, D. Area anomaly and generalized drift of iterated sums for hidden Markov walks. ArXiv Mathematics e-prints (2017). arXiv: 1709.04288.

Lopusanschi, O. and Simon, D. Lévy area with a drift as a renormalization limit of Markov chains on periodic graphs. Stochastic Process. Appl., 128 (7), 2404-2426 (2018). MR3804798.

Lyons, T. J. Differential equations driven by rough signals. Rev. Mat. Iberoamericana, 14 (2), 215-310 (1998). MR1654527.

Namba, R. A remark on a central limit theorem for non-symmetric random walks on crystal lattices. Math. J. Okayama Univ., 60, 109-135 (2018). MR3728433.

Orenshtein, T. Rough invariance principle for delayed regenerative processes. ArXiv Mathematics e-prints (2021). arXiv: 2101.05222.

Sabot, C. and Tournier, L. Random walks in Dirichlet environment: an overview. Ann. Fac. Sci. Toulouse Math. (6), 26 (2), 463-509 (2017). MR3640900.

Slutsky, E. Über stochastische Asymptoten und Grenzwerte. Metron Bd., 5, 3-89 (1925).

Sznitman, A.-S. Slowdown estimates and central limit theorem for random walks in random environment. J. Eur. Math. Soc. (JEMS), 2 (2), 93-143 (2000). MR1763302.

Sznitman, A.-S. On a class of transient random walks in random environment. Ann. Probab., 29 (2), 724-765 (2001). MR1849176.

Sznitman, A.-S. An effective criterion for ballistic behavior of random walks in random environment. Probab. Theory Related Fields, 122 (4), 509-544 (2002). MR1902189.

Sznitman, A.-S. and Zerner, M. A law of large numbers for random walks in random environment. Ann. Probab., 27 (4), 1851-1869 (1999). MR1742891.

Wong, E. and Zakai, M. On the convergence of ordinary integrals to stochastic integrals. Ann. Math. Statist., 36, 1560-1564 (1965). MR195142. 